\title{
The laser assisted field electron emission from carbon nanostructure
}

\author{
D. A. Lyashenko' ${ }^{1}$, Yu. P. Svirko $2,3^{*}$, M. I. Petrov ${ }^{2,4}$ and A. N. Obraztsov 2,3
}

\begin{abstract}
We present experimental and theoretical study of the femtosecond light-assisted field electron emission from nanocarbon films. We demonstrate that irradiation with intense femtosecond laser pulse allows one to achieve electron emission density of up to $13 \mathrm{nC} / \mathrm{cm}^{2}$ at a moderate applied static electric filed. The developed model well describes obtained experimental results and allows us to visualize physical mechanisms including heating of electron gas, multiphoton photoionization, and the space charge formation, which are responsible for the observed phenomena.
\end{abstract}

Keywords: Carbon nanostructures, Field emission, Thermionic emission, Ultrashort laser pulses

\section{Background}

The electronic sources based on laser assisted electron emission have found applications in different fields of science and technology including particle accelerators [1], free electron lasers [2], and modern photovoltaic systems [3]. The laser assisted electron emission has opened a way towards the time resolved electron microscopy $[4,5]$ because electrical gating and source control enable time resolution down to picoseconds, while using optical control enables creation of electron pulses with duration down to tens of femtoseconds [6]. Such dense and short electron bunches can resolve important problems in electron microscopy, X-ray microscopy, and time-resolved electron diffraction, which is of special interest for industry and science. However, development of such electron sources requires cathode materials, which are capable to provide a high density electron current and possess a high ray stability [7-9]. One of the most promising materials here are nanocarbon films (NCF) consisting of disordered graphene sheets [10] that demonstrated high efficiency of the field electron emission and durability with response to laser treatment. Owing to their unique structure and relative simplicity of the fabrication process, NCF are promising for

\footnotetext{
* Correspondence: yuri.svirko@uef.fi

${ }^{2}$ Department of Physics and Mathematics, University of Eastern Finland, Joensuu 80101, Finland

${ }^{3}$ Department of Physics, M. V. Lomonosov Moscow State University, Moscow 119991, Russia

Full list of author information is available at the end of the article
}

various applications in electronic devices. However, implementation of the NCF in practice implies getting more information on the electron emission mechanism. This paper reports experimental results and theoretical analysis aiming at the mechanism of the ultrashort laser pulse assisted electron emission from NCF.

\section{Methods}

The NCF was deposited on the n-doped silicon substrate by using chemical vapour deposition (CVD) of the hydrogen-methane gas mixture [10]. One can observe from Fig. 1 that the fabricated film is composed of multilayer graphene crystallites, which are 2-20 nm thick and have lateral size of $1-3 \mu \mathrm{m}$. The crystallites are oriented preferably perpendicularly to the substrate and separated from one another by $0.5-1 \mu \mathrm{m}$.

Electron emission measurements were performed in a diode configuration with flat cathode and anode electrodes. The cathode was composed of NCF film deposited on the $20 \times 20 \mathrm{~mm}^{2}$ n-doped $\mathrm{Si}$ substrate, which was attached to a glass plate with indium contact layer. The anode consisted of $25 \times 25 \mathrm{~mm}^{2}$ glass plate covered by a transparent indium tin oxide (ITO) layer. The transmittance of the anode was about $80 \%$ in the visual wavelength spectral range. The glass plates holding anode and cathode electrodes were glued to each other by a vacuum epoxy glue. The distance between surfaces of NCF (cathode) and ITO (anode) was fixed at $500 \mu \mathrm{m}$ by nonconductive spacers. The field emission threshold 


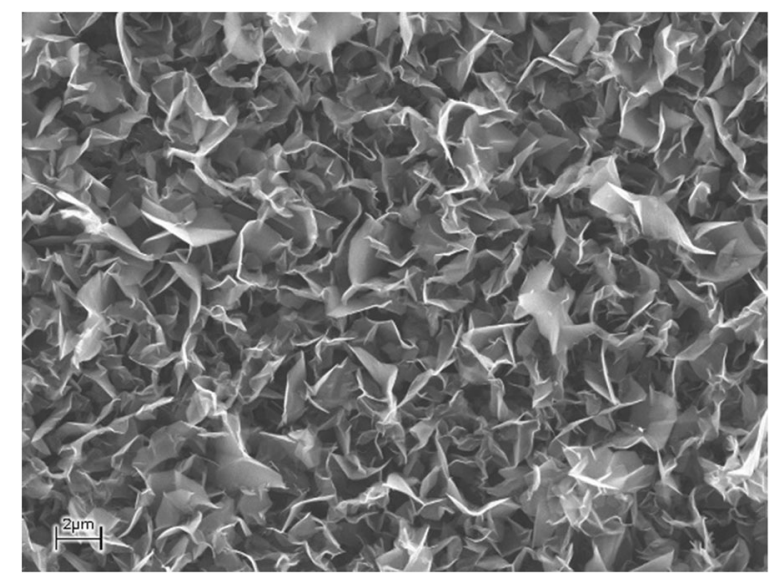

Fig. 1 The scanning electron microscope image of the NCF deposited on the silicon substrate

for NCF cathode was about $2 \mathrm{~V} / \mu \mathrm{m}$ [11], which corresponds to $1000 \mathrm{~V}$ threshold voltage. In our experiments, the applied voltage did not exceed $500 \mathrm{~V}$ to prevent the field emission influence on the measurements of the laser assisted emission.

In the laser-assisted electron emission experiment, we employ the femtosecond Ti:sapphire laser (CDP Tissa 50), which provided 50 fs pulses at $800 \mathrm{~nm}$ with repetition rate $50 \mathrm{~Hz}$ and the energy of up to $0.8 \mathrm{~mJ}$. The pulse energy was controlled by optical attenuators and measured by a power meter (Thorlabs). The laser beam diameter was fixed at $4 \mathrm{~mm}$ by an aperture. The second harmonic beam was produced by BBO crystal (labelled as SHG in Fig. 2). We performed laser assisted filed emission measurements at the angles of incidence of 90 and $45^{\circ}$ for the fundamental $(800 \mathrm{~nm})$ and the second harmonic $(400 \mathrm{~nm})$ beams, which were cross-linear polarized. The excitation wavelength was selected by aligned grid polarizers P1 and P3. The energy of the incident laser pulse was varied by rotating Glan polarizer P2. Beam splitter and fast photodiode PD were employed to control the incident pulse energy. The photodiode was calibrated by power meter for in-situ pulse energy measurement. The irradiated area on the cathode did not exceed $0.12 \mathrm{~cm}^{2}$ at normal incidence angle. The mutual orientation of the polarization azimuth of the incident beam and the cathode was varied by rotation the diode around substrate normal. In our experimental conditions, we did not observe any degradation of NCF film under irradiation with intense laser pulses. This indicates that energy density in the incident laser pulse was below the damage threshold.

\section{Results}

Figure 3 shows the dependence of the emitted charge on the energy of the incident pulse and applied voltage. One can observe from Fig. 3 that the laser assisted emission has pulse energy threshold, which corresponds to the switching of the emission mechanism from multiphoton to thermionic as the pulse energy increases. Under femtosecond excitation, the thermionic regime, which is related to the heating of electron gas by the light pulse, can be described in the framework of the two-temperature (electron-phonon) model.

The electron emission from the NCF cathode is suppressed by the volume charge, which is accumulated above the cathode surface. The volume charge manifests itself as a saturation-like dependence of the emitted charge on the laser pulse energy when the applied voltage is below $100 \mathrm{~V}$. However if the applied voltage is above $100 \mathrm{~V}$, the emitted charge is nearly linear function of the pulse energy. The dynamics of the electron emission under femtosecond excitation involves complicated charge redistribution. In particular, repulsion from the cloud of already emitted electrons results in the partial reabsorption of the emitted electrons by the NCF [12].

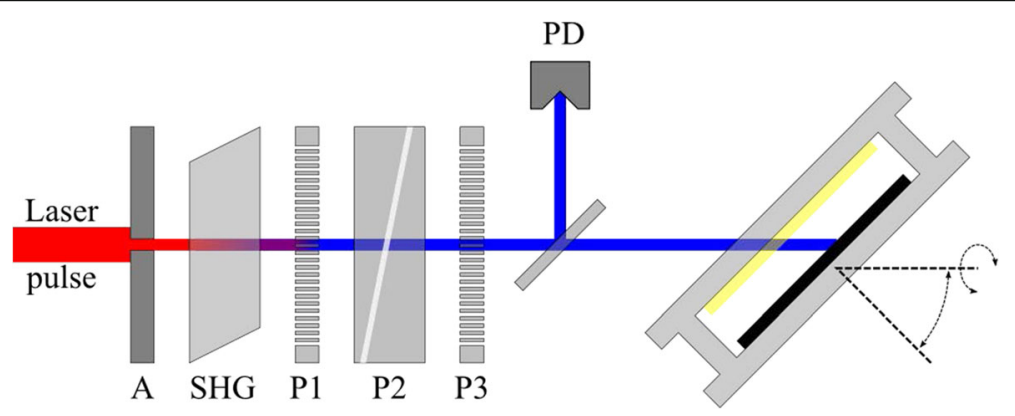

Fig. 2 Laser-assisted emission measurement setup. The $50 \mathrm{fs}$ long pulses at wavelength of 800 nm were provided by Tissa 50 Ti: sapphire oscillator and amplifier. Beam diameter was fixed to $4 \mathrm{~mm}$ by the aperture A. Nonlinear crystal SHG provided second harmonic pulses at the wavelength of $400 \mathrm{~nm}$. The fundamental and second harmonic beams are orthogonally polarized. The excitation pulse wavelength was selected by co-aligned grid polarizers P1 and P3. The energy of the incident pulse was controlled by the Glan polarizer P2. The energy of the incident pulse was measured by silicon photodiode PD, which was calibrated by a power meter for each wavelength and polarization of the incident pulse 


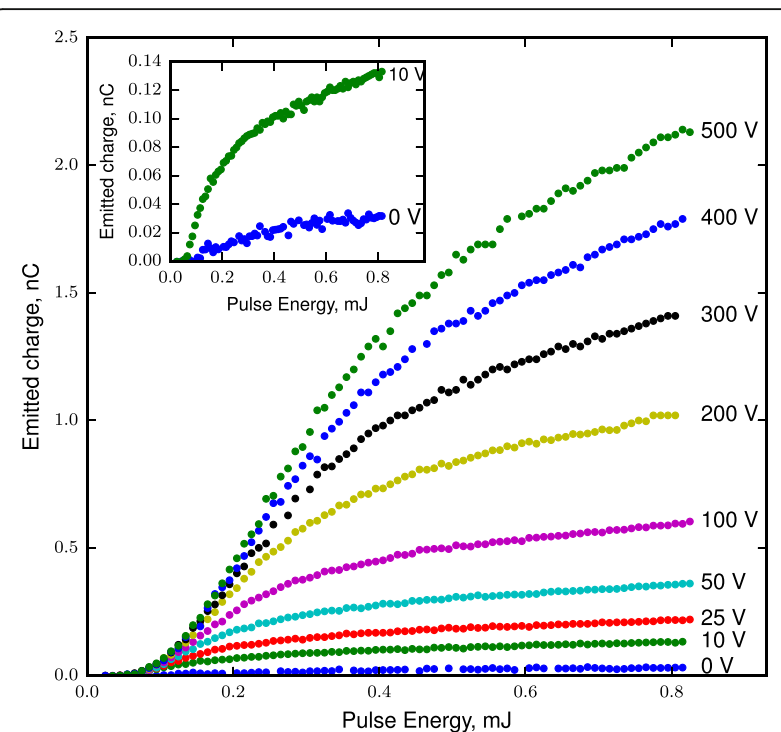

Fig. 3 The laser pulse energy dependence of the electric charge collected at the anode when the NCF cathode was irradiated by the fundamental $(800 \mathrm{~nm})$ beam. Inset shows the results of measurements at the applied voltage of 0 and $10 \mathrm{~V}$. The measurements were performed at normal incidence

One can observe from Fig. 3 that by varying the applied voltage and the laser pulse energy one can control the emitted charge from $0.03 \mathrm{nC}$ (it corresponds to the emission charge density of $0.24 \mathrm{nC} / \mathrm{cm}^{2}$ ) up to $1.6 \mathrm{nC}$ $\left(13 \mathrm{nC} / \mathrm{cm}^{2}\right)$.

From the polarization and wavelength dependence of the emitted charge shown in Fig. 4 one can observe that under normal incidence, the increase of photon energy hardly affects the emission efficiency (see Fig. 4c and d). This indicates that thermionic mechanism dominates emission above the threshold. Moreover, from Figs. 4a and $\mathrm{b}$ one can see that that increasing the photon energy shifts the threshold position, while the slope of the dependence of the emitted charge on the pulse power remains nearly the same also indicating the thermionic regime of the emission above the threshold.

\section{Discussion}

The obtained experimental results on the laser assisted emission from NCF can be analysed in the framework of the two-temperature model, which describes the observed phenomena in terms of coupling between electron and lattice systems of the graphite flakes that compose the NCF. Under irradiation of NCF with intense femtosecond pulses, the energy of photons is transfered to electrons creating an ensemble of hot electrons. In order to estimate the electron temperature we need to determine the free electron density, which can be considerably higher than its equilibrium value of about $10^{19} \mathrm{~cm}^{-3}$ [13].
The light absorption in graphite is determined by intraband and interband transitions. Under weak illumination, the interband absorption dominates due to relatively low concentration of free carries. The interband transitions lead to linear absorption coefficient of $\alpha_{\text {inter }}=5 \times 10^{5} \mathrm{~cm}^{-1}[14,15]$ and the free carriers photoexcitation rate of

$$
R_{\text {inter }}=\alpha_{\text {inter }} P / \hbar \omega
$$

where $P$ is the incident pulse intensity and $\hbar \omega$ is the photon energy.

The intraband absorption can be described in terms of the Drude model taking into account dependence of the plasma frequency $\omega_{p}$ on the free carrier concentration $N_{e l}$ [16]. In the Drude model framework, the dielectric permittivity is determined by the following equation:

$$
\varepsilon=\varepsilon^{\prime}+i \varepsilon^{\prime \prime}=\varepsilon_{\infty}-\frac{\omega_{p}^{2}\left(N_{e l}\right)}{\omega\left(\omega+\frac{i}{\tau}\right)}
$$

where $\varepsilon^{\prime}$ and $\varepsilon$ " are real and imaginary parts of dielectric permittivity, $\varepsilon_{\infty}=2.5$ and $\tau \approx 10^{-13} s$ [17] is the electron momentum relaxation rate. The plasma frequency is scaled with $N_{e l}$ as

$$
\omega_{p}\left(N_{e l}\right)=\omega_{p}\left(N_{e l}^{0}\right) \sqrt{\frac{N_{e l}}{N_{e l}^{0}}}
$$

where $N_{e l}^{0}$ is the equilibrium free carriers density. In equilibrium conditions, the plasma frequency for graphite can be estimated as $\hbar \omega_{p}\left(N_{e l}^{0}\right)=0.9 \mathrm{eV}$ is $[17,18]$. The intraband absorption coefficient can be presented in the following form:

$$
\alpha_{\text {intra }}=\frac{2 \omega}{c} \sqrt{\frac{-\varepsilon^{\prime}+\sqrt{\varepsilon^{\prime 2}+\varepsilon^{\prime \prime 2}}}{2}}
$$

The power density absorbed by electronic system of the NCF via inter- and intraband transitions is $Q_{a b s}=P\left(\alpha_{\text {inter }}\right.$ $\left.+\alpha_{\text {intra }}\right)$.

The temporal evolution of the electron ensemble temperature $T$ is described by the following heat transfer equation:

$$
C(T) \frac{\partial T}{\partial t}=C_{p h} \frac{T_{p h}-T}{\tau_{p h}}+Q_{a b s}
$$

where $C(T)$ and $C_{p h}$ are the electron and phonon heat capacitances, $T_{p h}$ is the lattice temperature, $\tau_{p h}$ is the rate of the energy transfer from electron ensemble to the lattice. The first term in the right-hand side of Eq. (5) describes the electron-phonon coupling, which can be very strong in graphene-like structures [19]. It has been recently shown that $\tau_{p h}$ can be comparable to laser pulse duration [20] resulting in fast cooling of electronic 

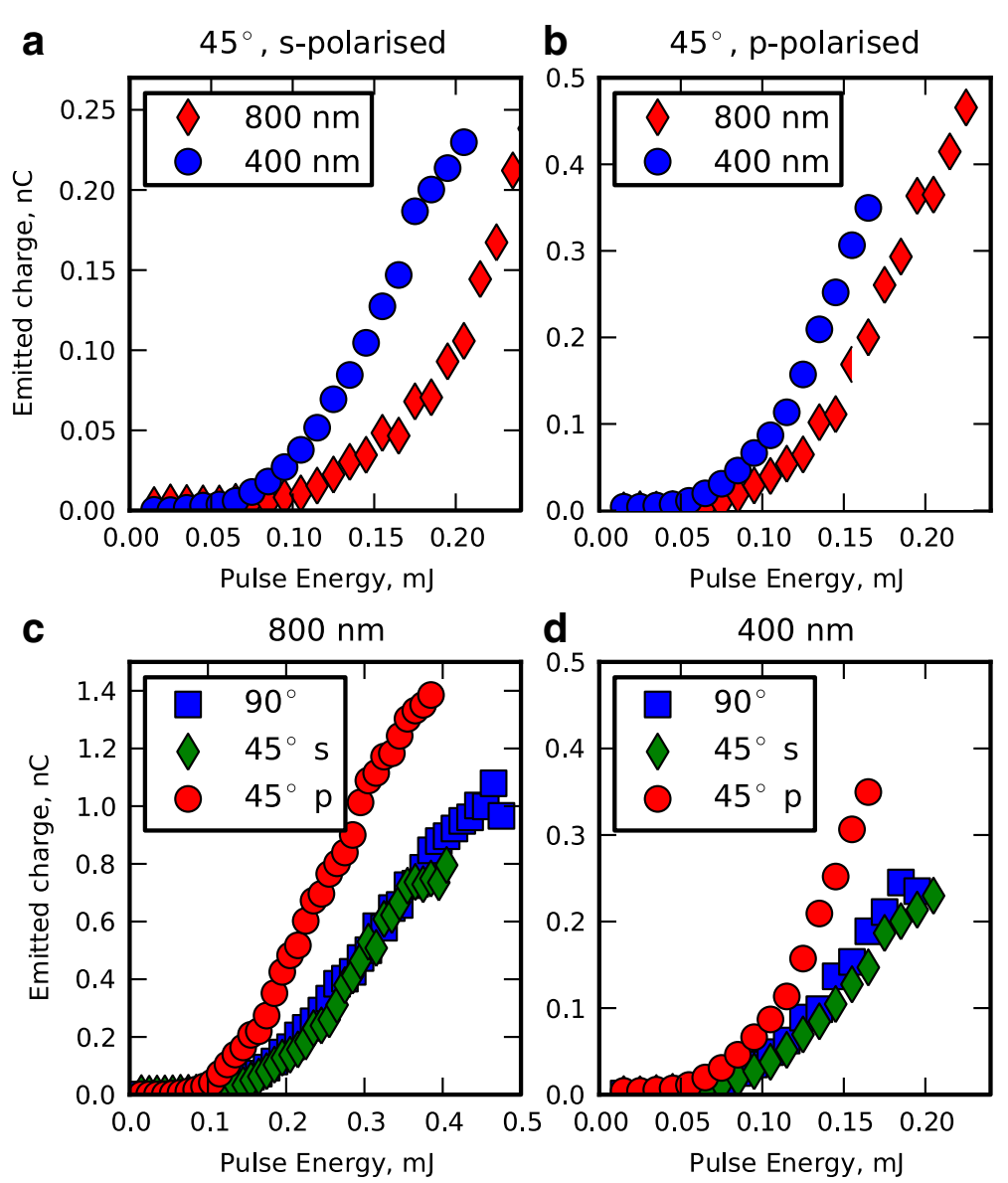

Fig. $4 \mathbf{a}$ and $\mathbf{b}$ The dependence of the emitted charge on the laser pulse energy for the s-and p-polarized laser beams, respectively, at the incident angle of $45^{\circ}$. $\mathbf{c}$ and $\mathbf{d}$ Emitted charge as a function

system [21]. In numerical modelling, we used $\tau_{p h} \approx 30 \mathrm{fs}$ $[20,22]$ assuming that phonon temperature stays constant during electron emission process due to large phonon heat capacitance.

The electron heat capacitance can be obtained from the following equation:

$$
C(T)=\int_{0}^{\infty} E D(E) \frac{\partial f(E, \mu(T))}{\partial T} d E
$$

where density of electronic states is $D(E)=D_{0}+D_{1} E, D_{0}=7$ $10^{21} \mathrm{eV}^{-1} \mathrm{~cm}^{-3}$ and $D_{1}=310^{21} \mathrm{eV}^{-2} \mathrm{~cm}^{-3}$ [23]. This model allows us to obtain temperature of the electron gas under femtosecond laser excitation.

The numerical simulation of the electron ensemble temperature using Eqs. $(5,6)$ shows (see Fig. 5) that the electron temperature increases up to $5500 \mathrm{~K}$ under irradiation of NCF with $0.75 \mathrm{~mJ}$ pulse. This result well corresponds to the electron heating in highly ordered pyrolytic graphite films reported in Ref. [21].

In order to take into account the space charge, which is formed at the laser pulse front [21, 24], we use approach [24], which describes the thermionic emission taking into account multiphoton processes. One can see from the Fig. 6 that the theory predicts a threshold-like dependence of emitted charge on the pulse energy in a good correspondence with experimental results. At low temperature (i.e. at a low pulse energy) we observe exponential growth of the emitted charge in accordance with the Richardson-Dushman equation. When the electron temperature exceeds $2500 \mathrm{~K}$, the electrostatic energy of space charge becomes higher than the thermal energy and the emission is suppressed by the space charge formation. As a result, the emitted charge shows nearly linear dependence on the electron temperature. The thermionic emission takes place when the laser pulse energy exceeds $0.1 \mathrm{~mJ}$ (see Fig. 6). Results of our experiment shown in Fig. 4 confirm that multiphoton photoemission dominates the electron yield [24] when the pulse energy is below $0.1 \mathrm{~mJ}$.

The threshold electron temperature corresponds to pulse energy of $0.1 \mathrm{~mJ}$ (see Fig. 6) when the switching the emission regime from multiphoton to thermionic occurs. At the laser pulse energy below the thermionic 


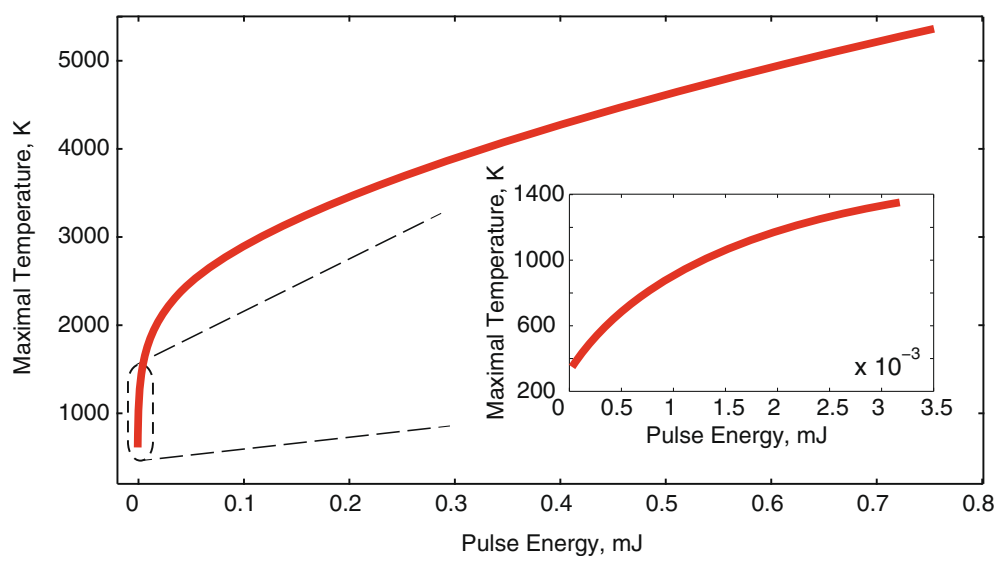

Fig. 5 The maximum electronic temperature of the in NCF as a function of the laser pulse energy at the pulse duration $50 \mathrm{fs}$ and the excitation wavelength of $800 \mathrm{~nm}$

threshold, the multiphoton photoemission [12] dominates (see also Fig. 4).

\section{Conclusion}

We performed experimental and theoretical study of the laser assisted electron emission from nanocarbon films. In the experiment, we demonstrate that the density of the electric charge collected at the anode of the vacuum diode with the NCF cathode can be as high as $13 \mathrm{nC}$ / $\mathrm{cm}^{2}$ under irradiation with laser pulse with energy of $0.75 \mathrm{~mJ}$ and duration of $80 \mathrm{fs}$ at the applied bias voltage of $500 \mathrm{~V}$. The dependence of the emitted charge on the applied voltage evidences the accumulation of spatial charge, which can suppress electron emission at a low bias. The obtained dependence of emission efficiency on excitation wavelength demonstrates the importance of multiphoton processes in the laser-assisted emission. By

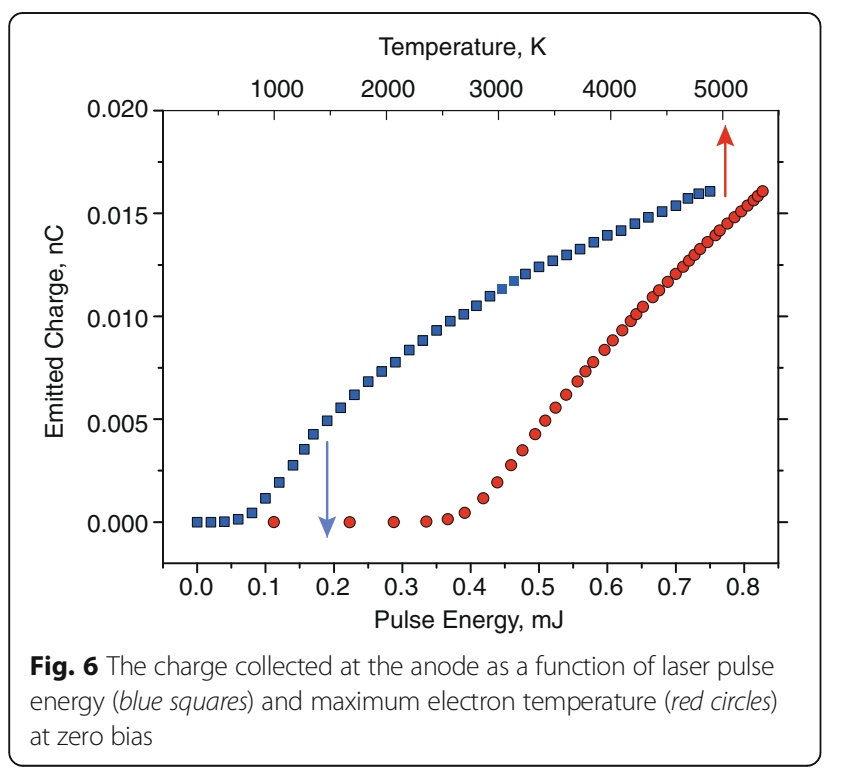

using results of the performed experiment, we developed the two-temperature model of the assisted emission that takes into account laser heating of the electron ensemble and energy exchange with the lattice. The model well corresponds to the experiment and can be used for development novel light-controlled sources based on nanocarbon cathodes.

\section{Abbreviations \\ CVD: Chemical Vapor Deposition; NCF: Nanocarbon films; SHG: Second harmonics generation}

\section{Funding}

This work was supported by the Russian Science Foundation (Grant No. 14-1200511), FP7 Marie Curie Program (Grant No. PIRSES-GA-2011-295241) and Academy of Finland (Grant No 298298).

\section{Authors' contributions}

Dr DAL carried out experiment on the laser-assisted electron emission. Dr MIP performed modeling of the electron emission and took part in the writing the manuscript. Dr ANO fabricated the nanocarbon cathodes and designed the diode. Dr YPS took part in the modeling of the electron emission and prepared the manuscript. All authors read and approved the final manuscript.

\section{Competing interests}

The authors declare that they have no competing interests.

\section{Author details}

${ }^{1}$ Texas State University, 601 University Drive, San Marcos, TX 78666, USA. 2Department of Physics and Mathematics, University of Eastern Finland, Joensuu 80101, Finland. ${ }^{3}$ Department of Physics, M. V. Lomonosov Moscow State University, Moscow 119991, Russia. ${ }^{4}$ ITMO University, Saint-Petersburg 199034, Russia.

Received: 25 November 2016 Accepted: 2 January 2017

Published online: 25 January 2017

\section{References}

1. Brogle, R., Muggli, P., Davis, P., Hairapetian, G., Joshi, C.: Studies of linear and nonlinear photoelectric emission for advanced accelerator applications. Proceedings of the Particle Accelerator Conference, p. 1039. (1995)

2. Kong, S.H., Kinross-Wright, J., Nguyen, D.C., Sheffield, R.L.: Photo cathodes for free electron lasers. Nucl. Instrum. Methods Phys. Res., Sect. A 358, 272 (1995)

3. Schwede, J.W., Bargatin, I., Riley, D.C., Hardin, B.E., Rosenthal, S.J., Sun, Y., Schmitt, F., Pianetta, P., Howe, R.T., Shen, Z.-X., Melosh, N.A.: Photonenhanced thermionic emission for solar concentrator systems. Nat. Mater. 9, 762 (2010) 
4. Barwick, B., Park, H.S., Kwon, O.-H., Spencer Baskin, J., Zewail, A.H.: 4D imaging of transient structures and mor phologies in ultrafast electron microscopy. Science 322, 1227 (2008)

5. Zewail, A.H.: 4D ultrafast electron diffraction, crystallography, and microscopy. Annu. Rev. Phys. Chem. 57, 65 (2006)

6. Mustonen, A., Beaud, P., Kirk, E., Feurer, T., Tsujino, S.: Efficient light coupling for optically excited high-density metallic nanotip arrays. Sci. Rep. 2, 916 (2012)

7. Swanwick, M.E., Keathley, P.D., Fallahi, A., Krogen, P.R., Laurent, G., Moses, J., Kärtner, F.X., Velásquez-García, L.F.: Nanostructured ultrafast silicon-tip optical field-emitter arrays. Nano Lett. 14, 5035-5043 (2014)

8. Dong, C.D., Swanwick, M.E., Keathley, P.D., Kaertner, F.X., Velasquez-Garcia, L.F. Multiplexing and scaling-down of nanostructured photon-triggered silicon field emitter arrays for maximum total electron yield. Nanotechnology 26, 265202 (2015)

9. Krüger, M., Schenk, M., Hommelhoff, P.: Attosecond control of electrons emitted from a nanoscale metal tip. Nature 475, 78-81 (2011)

10. Obraztsov, A.N., Zolotukhin, A.A., Ustinov, A.O., Volkov, A.P., Svirko, Y.P. Chemical vapor deposition of carbon films: in-situ plasma diagnostics. Carbon 41, 839 (2003)

11. Bandurin, D.A., Mingels, S., Kleshch, V.I., Lützenkirchen-Hecht, D., Müller, G., Obraztsov, A.N.: Field emission spectroscopy evidence for dual-barrier electron tunnelling in nanographite. Appl. Phys. Lett. 106, 233112 (2015)

12. Obraztsov, A.N., Volkov, A.P., Zakhidov, A.A., Lyashenko, D.A., Petrushenko, Y. V., Satanovskaya, O.P.: Field emission characteristics of nanostructured thin film carbon materials. Appl. Surf. Science. 215, 214 (2003)

13. Raman, R.K., Tao, Z., Han, T., Ruan, C.: Ultrafast imaging of photoelectron packets generated from graphite surface. Appl. Phys. Lett. 95, 181108 (2009)

14. Kuzmenko, A.B., van Heumen, E., Carbone, F., van der Marel, D.: Universal optical conductance of graphite. Phys. Rev. Lett. 100, 117401 (2008)

15. Johnson, L.G., Dresselhaus, G.: Optical properties of graphite. Phys. Rev. B 7, 2275 (1982)

16. Arndt, A., Spoddig, D., Esquinazi, P., Barzola-Quiquia, J., Dusari, S., Butz, T.: Electric carrier concentration in graphite: dependence of electrical resistivity and magnetoresistance on defect concentration. Phys. Rev. B 80, 195402 (2009)

17. Dyukin, R.V., Martsinovski, G.A.: Electrophysical phenomena accompanying femtosecond impacts of laser radiation on semiconductors. Laser Physics and Engineering. 78, 88 (2011)

18. Tatar, R.C., Rabii, S.: Electronic properties of graphite a unified theoretical study. Phys. Rev. B 25, 4126 (1982)

19. Kampfrath, T., Perfetti, L., Schapper, F., Frischkorn, C., Wolf, M.: Strongly coupled optical phonons in the ultrafast dynamics of the electronic energy and current relaxation in graphite. Phys. Rev. Lett. 95, 187403 (2005)

20. Butscher, S., Milde, F., Hirtschulz, M., Knorr, A.: Hot electron relaxation and phonon dynamics in graphene. Appl. Phys. Lett. 91, 203103 (2007)

21. Lui, C., Mak, K., Shan, J., Heinz, T.: Ultrafast photoluminescence from graphene. Phys. Rev. Lett. 105, 1 (2010)

22. Breusing, M., Ropers, C., Elsaesser, T.: Ultrafast carrier dynamics in graphite. Phys. Rev. Lett. 102, 1 (2009)

23. Seibert, K., Cho, G.C., Kutt, W., Kurz, H., Reitze, D.H., Dadap, J.l., Ahn, H., Downer, M.C., Malvezzi, A.M.: Femtosecond carrier dynamics in graphite. Phys. Rev. B 42, 2842 (1990)

24. Riffe, D.M., Wang, X.Y., Downer, M.C., Fisher, D.L., Tajima, T., Erskine, J.L., More, R. M.: Femtosecond thermionic emission from metals in the space-charge-limited regime. J. Opt. Soc. Am. B 10, 1424 (1993)

\section{Submit your manuscript to a SpringerOpen ${ }^{\circ}$ journal and benefit from:}

- Convenient online submission

- Rigorous peer review

- Immediate publication on acceptance

- Open access: articles freely available online

- High visibility within the field

- Retaining the copyright to your article 